\title{
Portable and wireless imaging of dorsal hand vein
}

\author{
See Hui Ern', Audrey Huong ${ }^{2}$, Wan Mahani Hafizah Wan Mahmud ${ }^{3}$, Xavier Ngu ${ }^{4}$ \\ ${ }^{1}$ Shenzhen PTY Medtech Co Ltd, A-27-13, Menara UOA Bangsar, Malaysia \\ ${ }^{2,3,4}$ Faculty of Electrical and Electronic Engineering, Universiti Tun Hussein Onn Malaysia, Malaysia
}

\begin{tabular}{l}
\hline \hline Article Info \\
\hline Article history: \\
Received Feb 2, 2020 \\
Revised Mar 6, 2020 \\
Accepted Mar 24, 2020 \\
\hline Keywords: \\
Body mass index \\
Imaging \\
Portable \\
Skin tone \\
Vein
\end{tabular}

\begin{abstract}
Technologies for visualization of dorsal hand vein are of the great interest in the studies of drugs-body response, identity authentication and human metabolism. This study integrates near infrared (NIR) technology into an optical system for non-contact, mobile and quick on the spot visualization of dorsal vascular system. The performance of the developed system was tested on twenty subjects of different skin tone and body frame dimensions. The results showed that the proposed system is able to produce output image of Signal-Noise Ratio (SNR) and Peak SNR of greater than $30 \mathrm{~dB}$ and $20 \mathrm{~dB}$, respectively. While this work found a correlation between skin tone and image quality metrics, high consistency was observed in the quality metrics calculated for image data of individuals having different body frame size. This work concluded that the proposed system can be suitably used as a portable and robust tool for enhanced visualization of dorsal hand vein.
\end{abstract}

Copyright $\odot 2020$ Institute of Advanced Engineering and Science. All rights reserved.

\section{Corresponding Author:}

Audrey Huong,

Faculty of Electrical and Electronic Engineering,

Universiti Tun Hussein Onn Malaysia,

86400 Batu Pahat, Johor, Malaysia.

Email: audrey@uthm.edu.my

\section{INTRODUCTION}

Blood vessel or vascular is an essential part of human circulatory system, which function is to carry blood through the body. There are two fundamental types of blood vessels namely arteries and veins. The arteries are to deliver oxygenated blood to the whole body whereas the veins return deoxygenated blood back to the heart. Among the well-connected vascular networks in the human body, study of vascular system in dorsal region of hands has received significant attentions in the past due to its apparent thin dermal layer where abundance of veins and arteries can be found [1]. While studies of dorsal arterial system present some controversies regarding the frequency and location of its artery branches [2], researches on dorsal hand vein were widely reported especially on that related to clinical venipuncture practices, in investigation of drugbody interactions [3] and metabolic studies [4]. In addition, the uniqueness and distinctive signatures of each individual's dorsal vascularity pattern making this an attractive alternative for biometric identification using palm print, retinal pattern, facial images and others [5-6].

Venipuncture is complex procedure; it is usually conducted when a hollow needle is poked through the skin into a superficial vein to draw blood during transfusion, for glucose and cholesterol monitoring, injection of drugs or liquid into the body, diagnosis of disease, etc. Conventionally, nurses who carry out these procedures would soak a cotton pad into alcohol and apply it onto the targeted injected area. Some assistants would also slap lightly on the selected site, grip and relax the associated arm for several times to stimulate the blood circulation prior to the venipuncture. A previous study [7] has shown that there is approximately $3 \%$ chance that a healthcare professional would be unable to locate vein in the first attempt. The major reason for this is due to poor vein visualization and tactile experience of young staff. Other factors include differences in individuals' skin thickness, size and depth of their veins, skin tone and body size. This group of individuals is known as Difficult Venous Access (DVA) patients [8]. The result often leads to 
multiple attempts, causing the puncture site to become reddish and swollen, bleeding in the vein, darkening of a vein due to peripheral venous scarring, and this could also result in permanent damage to the vein especially in pediatrics and elderly. Other side effects include physically discomfort, frightful experience and dissatisfaction. All these may lead to deferred treatment [9].

Previous systems used in the imaging of dorsal hand vein include X-Ray, ultrasound technique, multispectral imaging and thermal heating technology [10-11]. While X-ray, ultrasound and multispectral system are bulky and non-portable, the use of thermal heating approach causes discomfort to its users. NIR illumination technology in the wavelength of $700 \mathrm{~nm}$ to $900 \mathrm{~nm}$ has become a preferable choice in the imaging of dorsal hand vein [9]. This is due to its non-invasive characteristic, non-ionizing features and the ability to penetrate deep into the skin [12]. Several devices such as Vasculuminator, Accuvein, Veinviewer and Vuetek Scientific were invented since the last decades to enhance the viewing visibility of the peripheral venous using NIR technology. However, among the limitations of these devices include high cost, poor portability and complexity in its operation.

Technologies for visualization of dorsal hand vein are of the great interest in the studies of drugsbody response, identity authentication and human metabolism [13-14]. This research aims to integrate near infrared (NIR) technology and a Complementary Metal Oxide Semiconductor (CMOS) camera into an optical system for non-contact, mobile and quick on the spot visualization of dorsal vascular system. This study hypothesized that an individual's body build, frame size and skin tone would influence the quality of output image, wherein a darker skin tone and higher obesity status would produce a lower image quality.

\section{RESEARCH METHOD}

\subsection{Experimental system and subjects}

The employed system involved the use of NIR light emitters arranged in an annular pattern, which platform can be glided into a Wifi CMOS action camera (model no. SJ4000), for illumination of dorsal region of hands. While the illumination system comprises of five light emitting diodes (LEDs) emitting light of wavelength $850 \mathrm{~nm}$, the detection of signals reflected from the selected skin region was via the CMOS camera. The latter has a built-in infrared (IR) blocking filter, which was removed to achieve a better visualization of the vein.

Meanwhile an Android mobile application was created using MIT Apps Inventor for portable visualization of hand vein image using a mobile phone. The image acquired using the Wifi camera was sent wirelessly to a mobile phone, the image was then transferred to a computing unit for further processing via an Internet Protocol (IP) network. The image can be saved in both mobile phone and a local database in accordance with user's preference for secure storing of data for future use. An overview of the system architecture is shown in Figure 1. Figure 2 shows the user interface of the developed mobile application and an illustration on the example of recorded data in the local database.

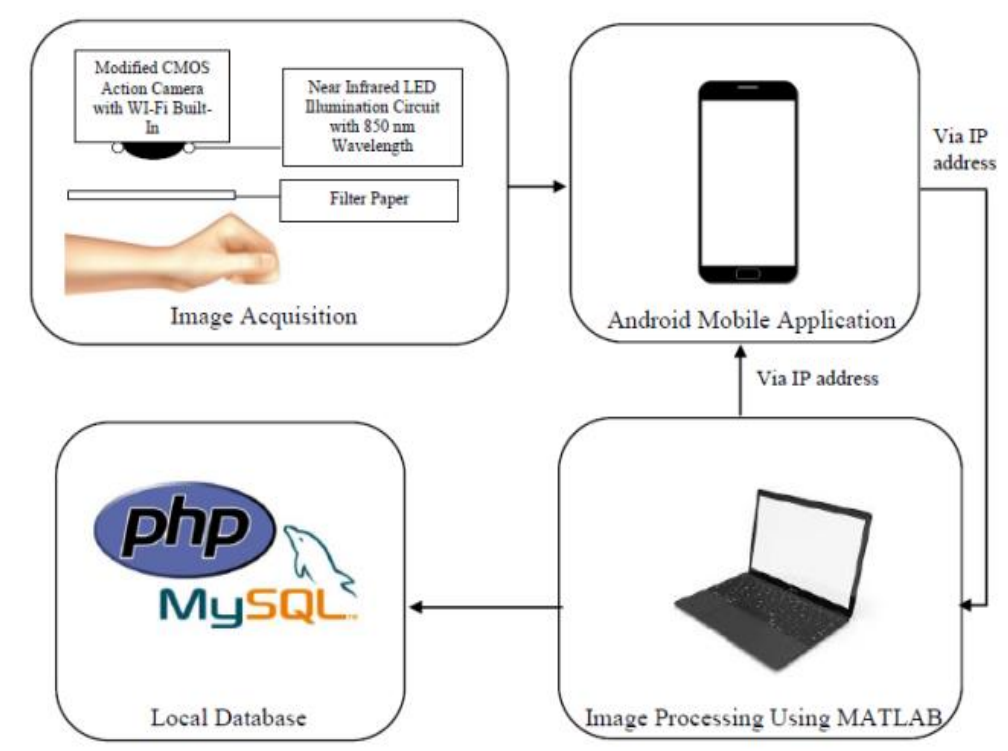

Figure 1. Overview of the system architecture 

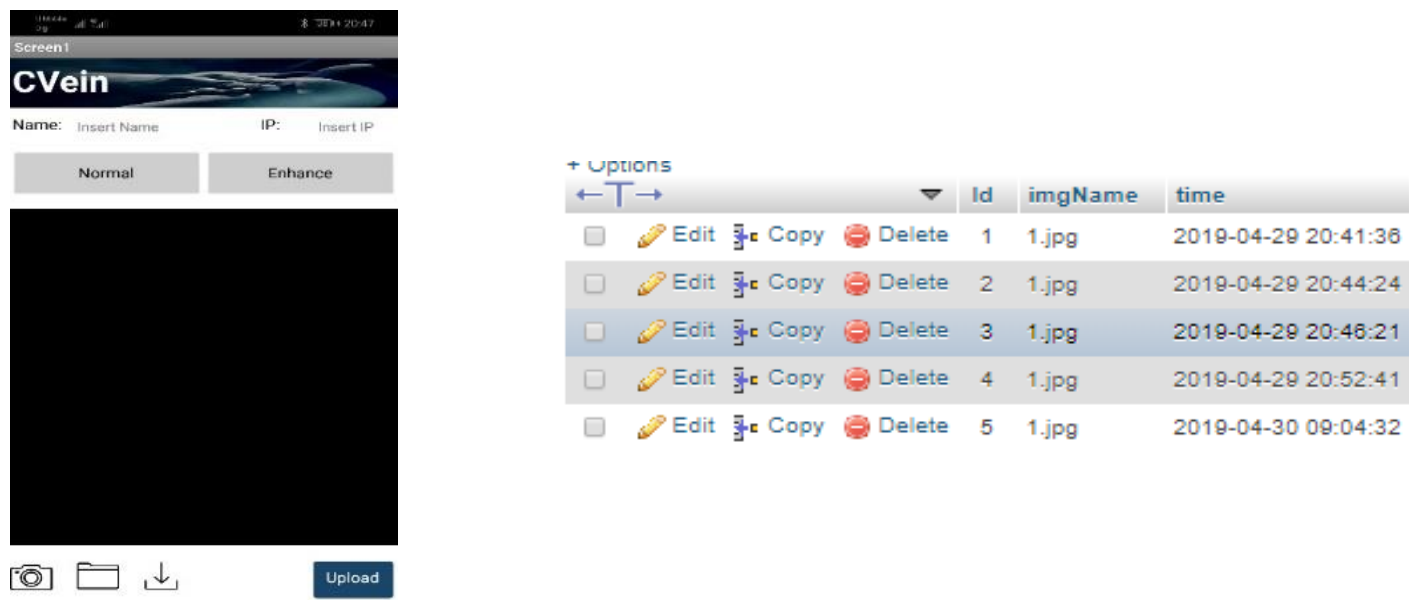

Figure 2. (Left) User interface of mobile application and (right) data records in a local database

The performance of this system was tested on twenty volunteers ( 12 females and 8 males) aged between 21 to 24 years. These subjects were students at Universiti Tun Hussein Onn Malaysia during the time of data collection. They all gave their signed informed consent to participate in this work. Since the visibility of veins may be influenced by a person's skin tone, physical build and condition, the body mass index (BMI) and skin tone (according to Fitzpatrick scale, i.e. skin type I-VI) of these participants were recorded. These participants were categorized into different groups according to their BMI and skin tone. The grouped subjects according to the calculated BMI included three underweight volunteers (BMI value < $18.5 \mathrm{~kg} / \mathrm{m}^{2}$ ), nine normal weight (BMI in between 18.5 to $24.5 \mathrm{~kg} / \mathrm{m}^{2}$ ), seven overweight (BMI range 25 and $29.9 \mathrm{~kg} / \mathrm{m}^{2}$ ) and one obese subject (BMI value $\geq 30 \mathrm{~kg} / \mathrm{m}^{2}$ ). Due to the limited subjects participates in this study, the skin tone of these subjects were grouped into three categories: skin type I and II, III and IV, and V and VI. There were 4, 7 and 11 subjects fall into these three categories, respectively.

\subsection{Image manipulation and processing}

The CMOS camera employed in this work is of 24-bit dynamic range, producing a Joint Photographic Experts Group (JPEG) image of resolution $4032 \times 3023$ pixels. The captured image was first cropped to select only region of interest of a dimension $2311 \times 2327$ pixels. The processing of the acquired image was via MATLAB (version R2017b) to enhance the visualization of vein pattern after image acquisition. The processing stage began with the color image being converted into grayscale image, representing the image in different shades of gray in between black and white, to reduce hue of the image that may lengthen the processing time [15]. The conversion of image from Red, Green, Blue (RGB) color space to 8-bit grayscale, $I$, was via the rgb2gray function.

Next, image enhancement was via Contrast Limited Adaptive Histogram Equalization (CLAHE), which is the modification from the original histogram. This technique divides an image into tiles or contextual region. A built in function (adapthisteq function) is available in MATLAB to implement contrast enhancement, which is arbitrarily chosen as factor 0.012. CLAHE was previously demonstrated in [16] to produce better image enhancement of low contrast medical images and improve the visibility of hidden features of the image by redistributing the used gray values. The contrast of the processed grayscale image was further adjusted (via imadjust function) using the limit range of $0.1-1$ to map intensity values in previous image to that in the new image.

\subsection{Image quality assessment}

The image quality is commonly evaluated using three main metrics (in unit decibel, $\mathrm{dB}$ ) namely Signal-to-Noise Ratio (SNR), Peak Signal-to-Noise Ratio (PSNR) and Mean Squared Error (MSE). SNR is a measure that compares the level of a desired signal (i.e. final output, $\left.I_{e}(x, y)\right)$ to the level of background noise given in (1).

$$
\mathrm{SNR}=\frac{I_{e}(x, y)}{\sqrt{M S E}}
$$

This parameter is often used to check the quality of image, wherein the value has to be greater than $30 \mathrm{~dB}$ to be qualified as a good image quality. There are correlation in between SNR and MSE; the latter 
parameter is inversely proportional to the SNR [11], it determines the average differences in the pixel between the original and the processed image [15] and is given by:

$$
M S E=\frac{\sum_{M, N}\left(I_{O}(x, y)-I_{e}(x, y)\right)^{2}}{M \times N}
$$

where $M, N$ is the dimension of image while $I_{0}(x, y)$ and $I_{e}(x, y)$ denote original grayscale and enhancedthreshold adjusted image, respectively, at pixel position $x$ and $y$. A low MSE value often indicates high similarity between the two images. Meanwhile the PSNR is used to calculate the ratio between the maximum possible signal power and the power of the distorting noise. The computed PSNR value may range from 30 to $50 \mathrm{~dB}$ for 8 bit data representation, wherein a high PSNR value represents a better image quality given by:

$$
P S N R=10 \log _{10} \frac{R^{2}}{M S E}
$$

where $R^{2}$ denotes peak value of maximal in an image data.

\section{RESULTS AND ANALYSIS}

An example of the acquired image cropped and converted into a grayscale range is shown in Figure 3. The image was further enhanced via CLAHE and undergone contrast adjustment. The resultant images are shown in Figure 4. This figure shows a significantly greater contrast in the dorsal hand veins as compared to the surrounding tissues and image background. Since veins appeared in a darker shade of gray (right of Figure 4), which eases the visualization process, image obtained from this step of the process was considered as the final output. The total image processing time was recorded as $34.8 \mathrm{~s}$. Meanwhile Figures 5-6 show examples of the processed image for individuals of different skin color and BMI.

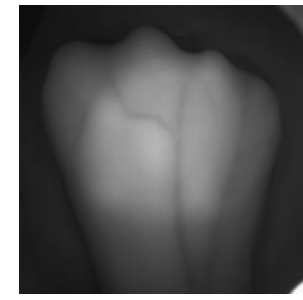

Figure 3. Image after cropped and grayscale conversio

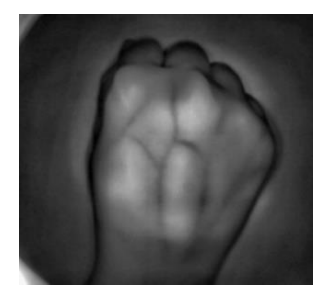

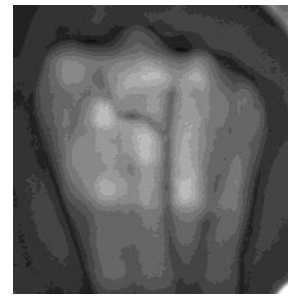

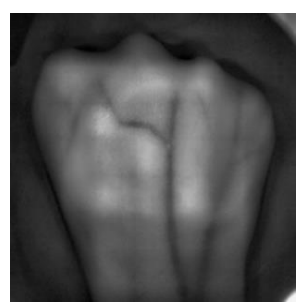

Figure 4. Processed image after (left) CLAHE enhancement and (right) contrast adjustment

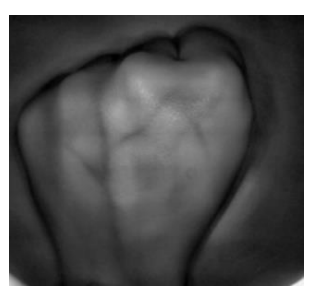

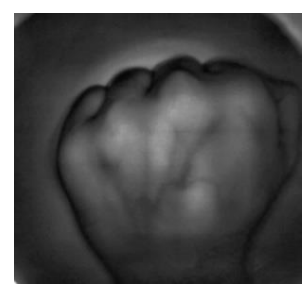

Figure 5. (From left to right) Processed image for individuals of skin color type I, III and V
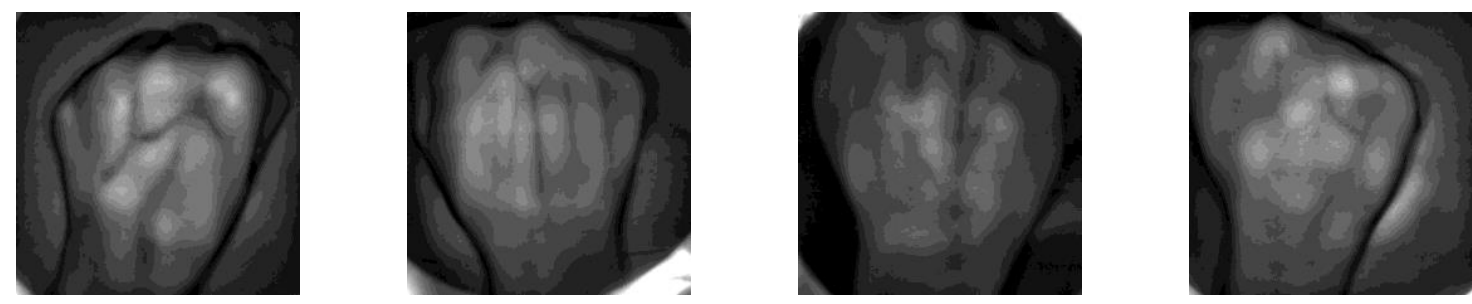

Figure 6. (From left to right) Processed image for individuals under categories underweight, normal weight, overweight and obese 
The mean and standard deviation (SD) of SNR, MSE and PSNR values calculated from the comparison between the grayscale image (for example that shown in Figure 3) and enhanced, threshold adjusted images (such as that shown in Figure 4) collected from the categorized skin tone groups discussed in section 2.1 are plotted and shown in Figure 7. Meanwhile Figure 8 shows the image quality metric values calculated for groups of different BMI.

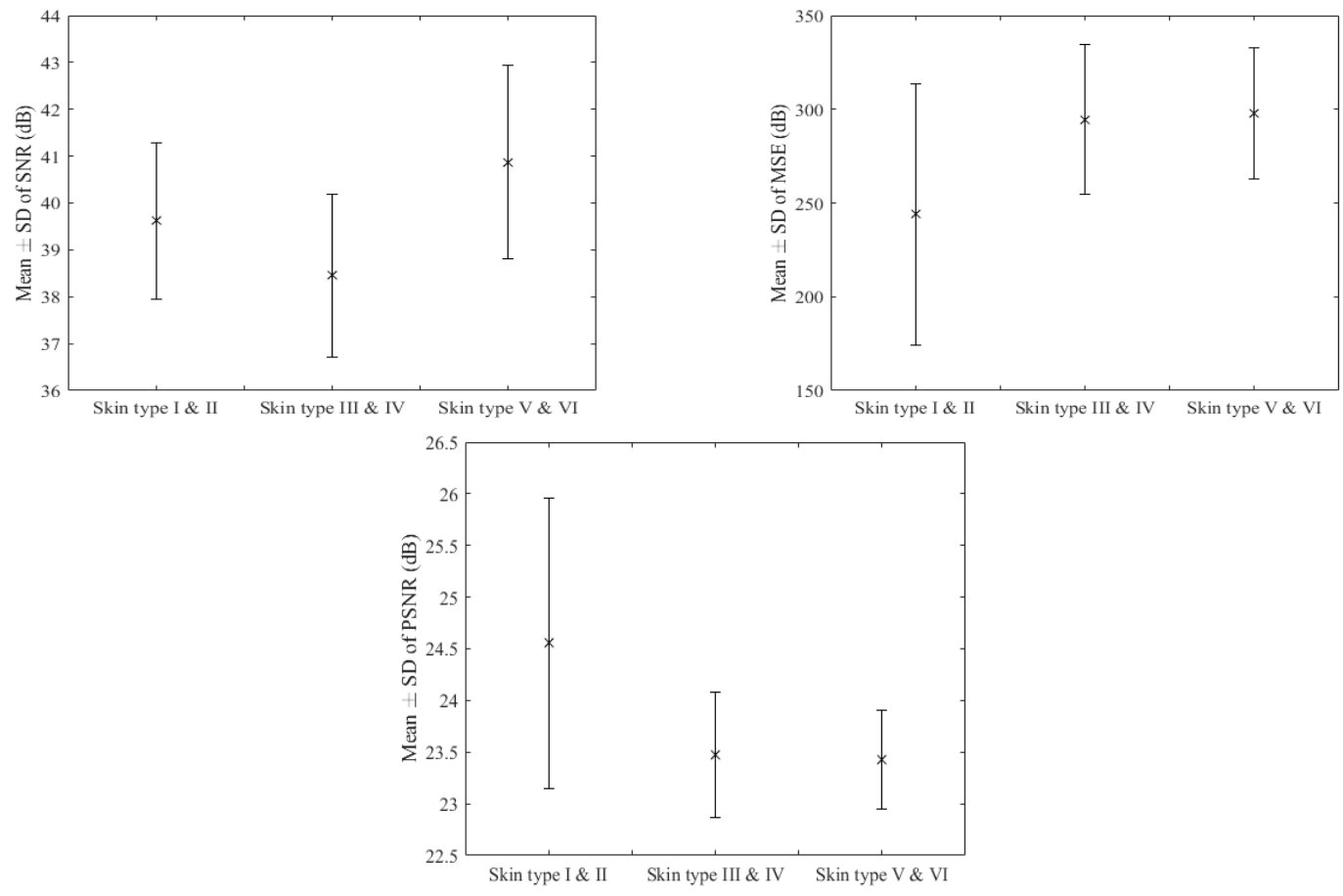

Figure 7. (From top to bottom) Mean and standard deviation (SD) of SNR, MSE and PSNR values calculated for the categorized skin tone (according to Fitzpatrik scale)
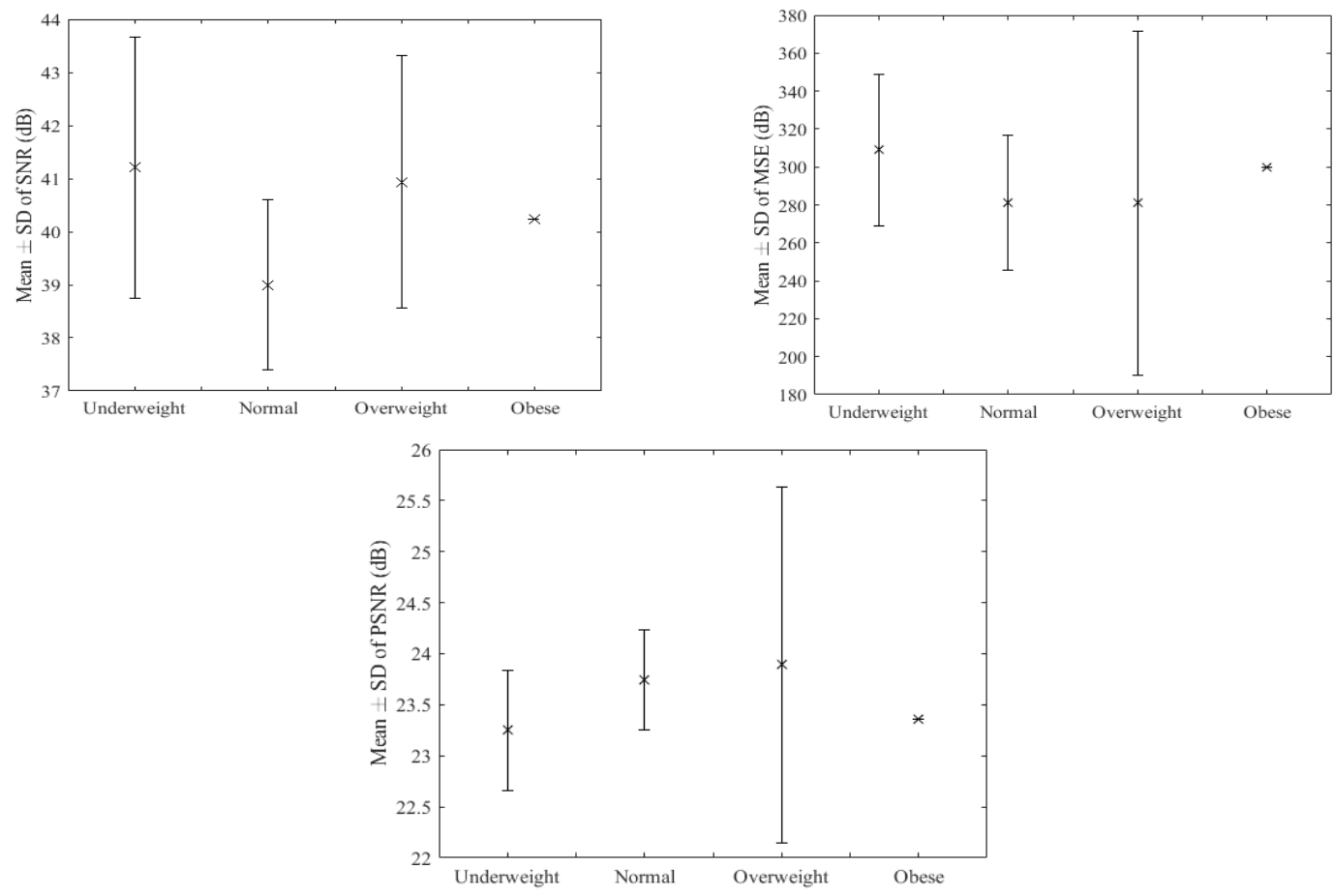

Figure 8. (From top to bottom) Mean and standard deviation (SD) of SNR, MSE and PSNR values calculated for the categorized BMI 


\section{DISCUSSION}

In this study attempts have been made to quantify the quality of dorsal hand image captured via the developed system with respect to the subjects' skin tone and BMI. Based on the results shown in Figures 7 and 8, it can be observed that even though the overall PSNR values were lower than that reported in [17], other work [18] suggested that 20 to $25 \mathrm{~dB}$ are accepted for image produced in wireless transmission. This is further supported by the recorded SNR, which revealed mean values greater than $30 \mathrm{~dB}$. Referring to Figure 7 the greatest PSNR values of $24.5 \pm 0.47 \mathrm{~dB}$ were collected for individuals of lighter skin color, while subjects of skin type III - VI showed slightly lower but similar results (the means are in the range of $23.42-23.47 \mathrm{~dB}$ ). This may be explained by the differences melanosome distribution, which may be found in abundance in darker skin type [19-20]. This light absorbing chromophore reduced the reflectance of light, rendering a lower signal intensity being detected [21]. This similar trend was observed in the computed MSE values, but in opposite manner. Interestingly the SNR results in Figure 7 showed that darker skin color has the highest SNR values $(40.8 \pm 2.06 \mathrm{~dB})$, followed by skin type I \& II. This finding indicated that the quality of processed images did not affected by skin tone of the recruited individuals, and visual wise, the veins can visibly be seen in skins of darker tones (type V\&VI). In addition, the considerably low SD values of SNR and PSNR metrics in the range of $0.47-2.06 \mathrm{~dB}$ in Figure 7 suggested relative good consistency in the quality of the output image for each skin type group in this study.

Unlike what we hypothesized in the beginning of this study, Figure 8 showed that groups of high BMI values do not necessary degrade image quality and affect visualization of veins. The results showed that individuals categorized as overweight and obese, and that underweight and normal weight produced similar PSNR and SNR values given by 23-24 dB and 39-41.5 dB range, respectively. This may be explained by the fact that BMI is a mere measurement of body fat (based on height and weight). Thus even though the strong correlation between subcutaneous adipose tissue thickness and a person's obesity status has been well established in the past [22-25], the authors believe some overweight individuals involved in this study may have thin adipose tissues in their hand. The differences in the integumentary system of the recruits can be deduced from the comparatively larger variation in the image quality metric (e.g. SD of PSNR of $1.74 \mathrm{~dB}$ ) calculated for the overweight group. It must be mentioned that Figure 8 showed a lower mean PSNR value of $23.2 \pm 0.58 \mathrm{~dB}$ for underweight individuals as compared to that of overweight subjects, an investigation into their skin type profile revealed that most of these subjects fall into the darker skin category. This further supports the possibility that the quality of the processed image and efficacy of vein visualization technology are influenced by a compound of different factors such as adipose tissue distribution, skin thickness and pigmentation, vein location and depth of an individual.

\section{CONCLUSION}

This work concluded that optical system with NIR ray of wavelength $850 \mathrm{~nm}$ produced reasonably good quality image for all skin colors and body frame dimensions, wherein the calculated SNR showed values greater than $30 \mathrm{~dB}$ while the PSNR values are within the acceptable limit for wireless transmission quality. This study found a correlation between skin tone and image quality metric where fair skins yielded the highest PSNR values. Meanwhile the performance of image quality metrics is considerably consistent for volunteers of different BMI. The results showed that the developed system may be suitably used for mobile and real time visualization of hand veins.

\section{ACKNOWLEDGEMENTS}

The authors are grateful for all volunteers participated in this work. This study is financially supported in part by Universiti Tun Hussein Onn Malaysia (under TIER 1 grant no. H223).

\section{REFERENCES}

[1] P. Oltulu, B. Ince, N. Kokbudak, S. Findik and F. Kilinc, "Measurement of epidermis, dermis and total skin thicknesses from six different body regions with new ethical histometric technique," Turkish Journal of Plastic Surgery, vol.26, pp. 56-61, 2018.

[2] M. Rezende, R. Junior, A. Cho, O. Hasegawa and S. Ribak, "Anatomic study of the dorsal arterial system of the hand," Rev Hosp Clin. Fac. Med. S. Paulo, vol. 59, pp. 71-76, 2004.

[3] A. Adefurin, et al., "Genetic variation in the alpha1B-adrenergic receptor and vascular response," The Pharmacogenomics Journal, vol. 17, pp. 366-371, 2017.

[4] A. Gorgey, G. Farkas, D. Dolbow, R. Khalil and D. Gater, "Gender dimorphism in central adiposity may explain metabolic dysfunction after spinal cord injury”, $P M \& R$, vol. 10, pp. 338-348, 2018. 
[5] J. Lee, T. Lo and C. Chang, "Dorsal hand vein recognition based on directional filter bank," Signal, image and video processing, vol. 10, pp. 145-152, 2016.

[6] C. Gopal, S. Srivastava, S. Bhardwaj and S. Bhargava, Applied Soft Computing, vol.47, pp. 12-20, 2016.

[7] F. Chandra, A. Wahyudianto andM. Yasin, "Design of vein finder with multi tuning wavelength using RGB LED," Journal of Physics: Conference Series, vol. 853, 2017.

[8] P. Anupongongarch, K. Khaosomboon and T. Keawgun, "Design and construction of median cubital vein transillumination device by using LED," IEEE Xplore, pp. 1-3, 2015.

[9] A. B. Bawase and M. S. D. Apte, "Infrared Hand Vein Detection System," IOSR Journal of Electronics and Communication Engineering, pp. 48-52, 2015.

[10] J. Fan, J. Yang, C. Wu, D. Ai, H. Song, A. Hao and Y. Wang, "Multiple features decomposition for subcutaneous vein extraction and measurement," IEEE Xplore, pp. 11265-11277, 2018.

[11] T. Walczak, J. K. Grabski, M. Michalowska and D. Szadkowska, "Application of artificial neural networks in the human identification based on thermal images of hands," Biomechanics in Medicine and Biology, vol. 831, pp. 114-122, 2019.

[12] M. Wadhwani, A. D. Sharma, A. Pillai, N. Pisai and M. Bhowmick, "Vein detection system using infrared light," International Journal of Scientific \& Engineering Research, vol. 6, pp. 780-785, 2015.

[13] B. M. Sontakke, V. T. Humbe and P. L. Yannawar, "Dorsal hand vein authentication system: A review," International Journal of Scientific Research Engineering and Technology, vol. 6, pp. 511-517, 2017.

[14] M. I. Delma, "The quest for Type 2 Diabetes subgroups identification: Literature review for a new subtype proposal," Cureus, vol. 10, pp. 1-6, 2018.

[15] B. M. Thomas, "Introduction to Video and Image Processing. Building Real System and Application," Denmark: Springer, 2012.

[16] B. B. Singh and S. Patel, "Efficient medical image enhancement using CLAHE enhancement and wavelet fusion," International Journal of Computer Applications, vol. 167, pp. 1-5, 2017.

[17] U. Sara, M. Akter and M. S. Uddin, "Image quality assessment through FSIM, SSIM, MSE and PSNR- a comparative study," Journal of Computer and Communication, Scientific Research Publishing, vol. 6, pp. 8-18, 2019.

[18] N. Thomos, N. Boulgouris and M. Strintzis, "Optimized transmission of jpeg2000 streams over wireless channels," Trans. Image Process., vol. 15, pp. 54-67, 2006.

[19] I. Hurbain, et al. "Melanosome distribution in keratinocytes in different skin types: Melanosome clusters are not degradative organelles," J Invest Dermatol., vol. 138, no. 3, pp. 647 - 656, 2018.

[20] T. Homma, S. Kageyama, A. Nishikawa, K. Nagata, "Melanosome degradation in epidermal keratinocytes related to lysosomal protease cathepsin V," Biochem. Biophys. Res. Commun., vol. 500, pp. 339-343, 2018.

[21] L. Souza-Barros, et al. "Skin color and tissue thickness effects on transmittance, reflectance, and skin temperature when using 635 and $808 \mathrm{~nm}$ lasers in low intensity therapeutics," Lasers Surg Med, vol. 50, no. 4, pp. 291-301, 2018.

[22] S. C. Yeon, K. H. Hyuck, J. K. Beom, N. K. Myeung and D. P. Hyo, "Development of a non-invasive measurement to the thickness of the subcutaneous adipose tissue layer," Exp. Dermatol., vol. 17, pp. 537-541, 20008.

[23] P. A. Fowler, M. F. Fuller, C. A. Glasbey, G. G. Cameron and M. A. Foster, "Validation of the in vivo measurement of adipose tissue by magnetic resonance imaging of lean and obese pigs," Am J Clin Nutr., vol. 56, no. 1, pp. 7-13, 1992.

[24] J. Zhang, H. He and A.F. Liu, "Identification of muscle and adipose gene expression patterns in lean and obese pig," S Afr J Anim Sc. vol. 49, no. 1, pp. 71-79, 2019.

[25] H. M. Aitken-Buck, et al. "Relationship between epicardial adipose tissue thickness and epicardial adipocyte size with increasing body mass index," Adipocyte, vol. 8, np. 1, pp. 412-420, 2019.

\section{BIOGRAPHIES OF AUTHORS}

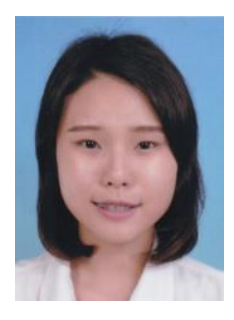

Hui Ern See received her bachelor degree in biomedical engineering from Universiti Tun Hussein Onn Malaysia. She is currently working as a virtual surgical design engineer and her research interests include orthognathic, reconstructive and temporomandibular joint surgeries for oral, and maxillofacial deformities.

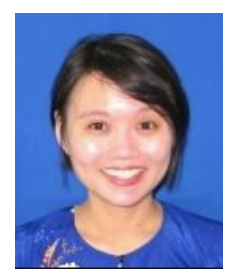

Audrey Huong is a senior researcher and Associate Professor at Universiti Tun Hussein Onn Malaysia. Her research interests include noninvasive physiology monitoring of human health, technology development and artificial intelligence assisted clinical diagnosis. 


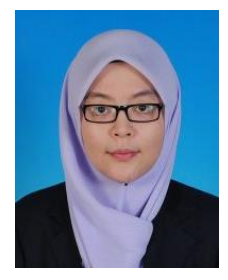

Wan Mahani Hafizah was born in Kuala Lumpur, Malaysia in 1987. She received her B.E. degree in biomedical engineering from Universiti Teknologi Malaysia, Malaysia, in 2009, and later her $\mathrm{PhD}$ degree in biomedical engineering from the same university in 2013. In 2014, she started joining the Department of Electronic Engineering, Universiti Tun Hussein Onn Malaysia, as a lecturer. Her current research interests include ultrasound imaging, medical image processing, and computer aided system, as well as telemedicine and rehabilitation.

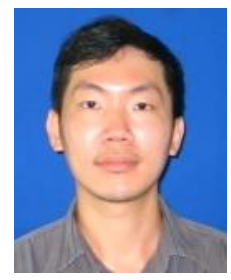

Xavier Ngu is a senior lecturer at Universiti Tun Hussein Onn Malaysia. His research interests include electrostatic discharge protection, electromagnetic compatibility and biomedical imaging. 